\title{
Parâmetros de fluência e tipos de erros na leitura de escolares com indicação de dificuldades para ler e escrever
}

\author{
Fluency parameters and types of errors in the reading of \\ students with signs of reading and writing difficulties
}

\author{
Cinthya Eiko Kawano', Adriana de Souza Batista Kida², Carolina Alves Ferreira Carvalho ${ }^{3}$, \\ Clara Regina Brandão de Ávila ${ }^{4}$
}

\begin{abstract}
RESUMO
Objetivo: Caracterizar o desempenho de escolares com indicação de dificuldades de leitura e escrita, segundo o ano escolar, categorias de erros, parâmetros de fluência leitora e as correlações entre essas variáveis. Métodos: Foram avaliadas 60 crianças (48\% meninas), do $3^{\circ}$ ao $5^{\circ}$ ano do Ensino Fundamental da rede pública. Trinta (dez de cada ano) que apresentavam indícios de dificuldades relacionadas ao aprendizado ou desempenho de leitura e escrita, compuseram o Grupo Pesquisa. Trinta, pareadas por idade e ano escolar, indicadas pelos professores como boas leitoras, compuseram o Grupo Controle. Todas leram, oralmente, duas listas de itens isolados (38 palavras e 29 pseudopalavras) e um texto. As leituras foram gravadas, transcritas e os parâmetros e erros, analisados. Resultados: Foram encontradas diferenças entre os grupos, com pior desempenho do Grupo Pesquisa em todas as variáveis estudadas. Os tipos de erros de leitura: desrespeito à regra de correspondência independente do contexto, omissões e adições, desrespeito à acentuação, erros complexos e recusas foram mais frequentes nesse grupo. As taxas e valores de fluência mostraram-se mais baixos nos escolares com queixas em relação aos bons leitores. Correlações negativas foram identificadas entre as variáveis de fluência de leitura e os diferentes erros, com diferentes valores para cada grupo e mostraram nessa amostra de escolares, que o número total de erros diminuiu com a progressão da escolaridade. Conclusão: Os escolares com indicação de dificuldades de leitura e de escrita apresentaram piores desempenhos de fluência na leitura, e maior número de erros em todos os anos escolares estudados. As correlações encontradas evidenciaram a influência do tipo de erro sobre a fluência da leitura, segundo diferentes padrões para cada grupo.
\end{abstract}

Descritores: Avaliação; Dislexia; Escolaridade; Transtornos de aprendizagem; Compreensão

\section{INTRODUÇÃO}

No que diz respeito à avaliação de leitura, o Programa Internacional de Avaliação de Alunos (PISA) ${ }^{(1)}$ procura verificar a operacionalização de esquemas cognitivos de leitura, pré-requisito básico para que os aprendizes possam continuar seus estudos de forma autônoma. Os resultados brutos para os países participantes indicaram na última avaliação, que o pior resultado para leitura foi o do Brasil com média de 393 pontos. Em dimensão numericamente expressiva, escolares

Trabalho realizado no Curso de Fonoaudiologia, Universidade Federal de São Paulo - UNIFESP - São Paulo (SP), Brasil.

(1) Curso de Graduação em Fonoaudiologia, Universidade Federal de São Paulo - UNIFESP - São Paulo (SP), Brasil.

(2) Universidade Federal de São Paulo - UNIFESP - São Paulo (SP), Brasil.

(3) Universidade Federal de São Paulo - UNIFESP - São Paulo (SP), Brasil.

(4) Departamento de Fonoaudiologia, Universidade Federal de São Paulo -UNIFESP - São Paulo (SP), Brasil.

Endereço para correspondência: Cinthya Eiko Kawano. R. Denis Antônio Casemiro, 21, Vila Rio Branco, São Paulo (SP), Brasil, CEP: 03875-020.

E-mail: eiko_kawano@hotmail.com

Recebido em: 26/10/2009; Aceito em: 21/4/2010 brasileiros, por diferentes motivos, fracassam no aprendizado acadêmico, e este fracasso tem sido mais e mais relacionado, às diferentes habilidades compreendidas na leitura.

Há vários mecanismos subjacentes à leitura proficiente, que conduzem à adequada compreensão do texto. Dentre estes, os iniciais, de decodificação, são fundamentais para o reconhecimento automático de palavras que permite o funcionamento pleno das funções de alta ordem implicadas na compreensão do texto lido ${ }^{(2-4)}$.

A maioria das pesquisas aponta que dificuldades, alterações ou distúrbios da leitura podem advir de déficits na decodificação e/ou de linguagem, estes afeitos às questões da compreensão ${ }^{(3-6)}$.

Os problemas de decodificação são mais frequentes e mais facilmente identificáveis no início do aprendizado escolar quando a fluência de leitura é o principal parâmetro de avaliação da competência leitora ${ }^{(7,8)}$. Contudo, a mensuração da taxa (número de palavras lidas por minuto - p.p.m.) e da acurácia (número de palavras lidas corretamente por minuto - p.c.p.m.) não são os únicos parâmetros reconhecidamente capazes de caracterizar a fluência da leitura. Pesquisas mais 
recentes têm estudado, objetivamente, aspectos prosódicos temporais na leitura ${ }^{(9,10)}$.

A indicação de que a leitura fluente depende do funcionamento harmonioso de diferentes capacidades e habilidades, inclusive as ligadas ao desempenho semântico, mostra que avaliar a fluência leitora não é tarefa simples ${ }^{(2,11)}$. Entretanto, parâmetros de taxa e de acurácia de leitura são ainda os mais investigados e utilizados, na avaliação de desempenho no contexto acadêmico ou clínico ${ }^{(12,13)}$. Estas medidas não devem ser analisadas de forma estanque, mas, interpretadas como componentes e auxiliares para o entendimento do aprendizado típico ou alterado, e do funcionamento normal ou deficitário de mecanismos e processos ligados à competência leitora.

$\mathrm{O}$ valor da acurácia com que as palavras são reconhecidas reflete o número de palavras lidas com exatidão. Assim, tanto menor seu valor quanto maior o número de incorreções produzidas no reconhecimento de palavras. Estas imprecisões podem também, influenciar os demais parâmetros da fluência $^{(14,15)}$. A análise do tipo de erro produzido pelo escolar não é frequente, o que leva o investigador a estabelecer algumas questões, como: o estudo do tipo de erro ou imprecisão leitora pode sinalizar as estratégias que o aprendiz utiliza na tentativa de ler corretamente? Ou, em que medida, a tentativa ou o fracasso de ler corretamente podem estar relacionados aos prejuízos da fluência?

Para que estes parâmetros de fluência e de categorias de erros de leitura pudessem ser estudados, nesta pesquisa solicitouse, a professores de duas escolas da Rede Pública de Ensino, que indicassem escolares que, comparativamente a seus pares de mesmo ano escolar, apresentassem dificuldades manifestas no aprendizado ou, no desempenho de leitura e escrita. Esses escolares não foram clinicamente avaliados, nem se consideraram informações referentes à possível participação em programas de recuperação ou terapêuticos. Buscou-se, dessa forma constituir uma amostra heterogênea de escolares, com indicações de dificuldades no aprendizado, sem levar em conta qualquer avaliação diagnóstica. Esses escolares, hoje, mais propensos ao fracasso escolar, dentro de alguns anos poderão compor um grupo a ser avaliado no PISA. A hipótese de que apresentem valores inferiores dos parâmetros de fluência e análise de erros, quando comparados a seus pares de mesma idade e ano escolar apontados, já no início da escolaridade, é admissível e já foi, inúmeras vezes, expressa na literatura ${ }^{(3,7,16-23)}$. Por outro lado, estudos preocupados com o desenvolvimento do aprendizado mostram resultados e evidências de que, mesmo na presença de dificuldades ou alterações, o desempenho em leitura e em escrita pode melhorar ${ }^{(3,24)}$.

O objetivo desta pesquisa foi de caracterizar um grupo de escolares indicados por apresentarem indícios de dificuldades no aprendizado ou desempenho de leitura e escrita, segundo o ano escolar, as categorias de erros e os parâmetros de fluência produzidos, além da presença de correlação entre essas variáveis.

\section{MÉTODOS}

Esta pesquisa foi aprovada pelo Comitê de Ética em da Universidade Federal de São Paulo - CEP/UNIFESP (nº 1490/08).

\section{Seleção da amostra}

Selecionou-se escolares regularmente matriculados do $3^{\circ}$ ao $5^{\circ}$ ano do Ensino Fundamental de duas escolas da Rede Estadual de Ensino, localizadas nas zonas Sul e Oeste do município de São Paulo, indicados por seus professores por apresentarem indícios de dificuldades no aprendizado ou de desempenho da leitura e da escrita e de aproveitamento escolar.

O Grupo Pesquisa (GP) foi constituído de 30 escolares (11 meninas), com indicação de dificuldade de leitura e escrita, sub-agrupados em: GPI - dez escolares do $3^{\circ}$ ano (sete meninos e três meninas, média de idade: 102 meses), GPII - dez escolares do $4^{\circ}$ ano (quatro meninos e seis meninas, média de idade: 116,7 meses) GPIII - dez escolares do $5^{\circ}$ ano (oito meninos e duas meninas, média de idade: 128,4 meses).

Para a composição do Grupo de Comparação (GC) utilizouse neste estudo dados de pesquisa anterior ${ }^{(25)}$ (aprovada pelo CEP, sob n $\left.{ }^{\circ} .1111 / 05\right)$, referentes às avaliações de 30 escolares típicos de $3^{\circ}$ a $5^{\circ}$ ano, considerados de bom aproveitamento escolar, por seus professores, sendo dez participantes de cada ano, pareados um a um, na proporção 1:1, aos escolares do GP segundo as faixas de escolaridade e de idade. Foram também sub-agrupados em GCI (seis meninos e quatro meninas, média de idade 103 meses), GCII (cinco meninos e cinco meninas, média de idade: 116,5 meses), GCIII (um menino e nove meninas, média de idade: 134,5 meses), segundo o ano escolar ao qual pertenciam.

Para a constituição dos grupos, solicitou-se aos professores a indicação de escolares que atendessem aos critérios gerais de inclusão na amostra: ausência de queixas relacionadas ou de indicadores de alterações da audição; ausência de queixas relacionadas ou de indicadores de alterações da visão (não corrigidos); ausência de queixas relacionadas ou de indicadores da presença de distúrbios neurológicos, comportamentais ou cognitivos; ausência de queixas ou indicadores de alterações de fala e de linguagem. Dentre os critérios especificamente definidos para a composição do GP estiveram: indicação somente de alunos com dificuldades manifestas no aprendizado da leitura e da escrita com consequentes dificuldades de aproveitamento escolar sem histórico de retenção, escrita, pelo menos, em nível alfabético e de reconhecimento de palavras escritas com regras de correspondência fono-grafêmica independente do contexto.

As avaliações tiveram início após o recebimento do TCLE, assinado pelos pais ou responsáveis das crianças indicadas. Os prontuários do GC também apresentaram os TCLE assinados.

Os pais e professores das crianças, do GP, cujas dificuldades no aprendizado da leitura e da escrita foram confirmadas, receberam orientação sobre a importância da avaliação fonoaudiológica completa e endereços de locais, no município, nos quais poderia encontrar serviço fonoaudiológico disponível. O mesmo procedimento de orientação e encaminhamento foi adotado no estudo anterior com as crianças sem dificuldades aparentes, mas que falharam na triagem realizada como procedimento de seleção da amostra. 


\section{Procedimentos}

\section{Coleta de dados}

Para a investigação dos parâmetros de fluência e de tipos de erros realizaram-se duas provas de leitura oral: a de itens isolados e a de textos.

Utilizou-se duas listas de itens isolados, sendo uma composta por 38 palavras (bota, laço, povo, bife, apito, fava, ganso, valsa, salame, mesada, deusa, carro, crescer, cenoura, cravo, ciclo, esgoto, assistir, depôs, fiz, texto, exagero, deixa, gente, gilete, alguém, sagu, joelho, guerra, hífen, lâmpada, ênfase, calma, sagui, xale, próximo, oxítona, absoluto.) e outra por 29 pseudopalavras (merra - chafo - cancha - taçã - fônus - xolher - caupa - fescer - gresco - galé - solota geleza - esbada -pelaço - contaso - absimpo - massafem - besego - vacoura - cambujão dau - gelhe - fuvo - morja - lirra -savocá - cinhela - jaino sefédu - gebeso), balanceadas quanto à extensão, frequência e ortografia ${ }^{(19,25)}$. As listas foram apresentadas impressas em folha A4 branca, em fonte Arial 16, dispostas verticalmente em espaçamento duplo.

Utilizou-se três textos, um para cada ano escolar, retirados de materiais didáticos apropriados: "O jabuti e a onça" para o $3^{\circ}$ ano; "O veterinário maluco" para o $4^{\circ}$ ano; "A coruja e a águia”, para o $5^{\circ}$ ano ${ }^{(23,25)}$. Os textos foram apresentados impressos em fonte Arial 12, com espaçamento 1,5.

Todos os escolares foram avaliados individualmente em uma única sessão, realizada em sala cedida pela direção da escola, em tempo que variou de dez a quinze minutos, sempre em horários de aula, previamente estabelecidos pela coordenação e equipe docente. Durante as sessões de avaliação a criança manteve-se sentada à mesa ou em carteira, posicionada de frente para a avaliadora. Adotou-se como condição para a avaliação a presença de ruídos em níveis que não interferissem na detecção da voz e na compreensão da mensagem oralmente apresentada pela examinadora. $\mathrm{Na}$ ausência de tais condições, a sessão foi interrompida e retomada posteriormente. Para a coleta do GP, seguiram-se os procedimentos definidos para a coleta do GC.

As leituras orais foram gravadas (em gravador digital marca Sony®) para análise posterior. Não foi estabelecido tempo limite e o acompanhamento com apoio digital durante a leitura não foi restringido.

\section{Análise dos dados}

Para a obtenção dos parâmetros de fluência de leitura transcreveu-se as gravações referentes às leituras orais. Mensurou-se o tempo despendido na leitura de cada lista ou texto e identificou-se os estímulos não lidos (recusas).

Para quantificação do desempenho calculou-se: a taxa (número de palavras lidas por minuto - p.p.m.) de leitura de palavras, apresentadas isoladamente ou as do texto e a de pseudopalavras, lidas por minuto a partir da seguinte fórmula:

Taxa $=\frac{n^{\circ} \text { de palavras ou pseudopalavras lidas X } 60 \text { segundos }}{\text { tempo total de leitura (em segundos) }}$

Para cálculo da acurácia computou-se, inicialmente, o número de palavras, ou de pseudopalavras, lidas corretamente por minuto. Considerou-se acerto somente quando os itens foram lidos correta e fluentemente. Ou seja, hesitações, estratégias de revisão para correções ou falhas na decodificação ortográfica foram consideradas erros. O cálculo da acurácia foi realizado a partir da seguinte fórmula:

Acurácia $=\frac{\mathrm{n}^{\text {o }} \text { de palavras ou pseudopalavras lidas corretamente X } 60 \text { segundos }}{\text { tempo total de leitura dos itens (em segundos) }}$

A obtenção dos valores de taxa na leitura do texto envolveu o cálculo do tempo total despendido na leitura do texto e a identificação das palavras omitidas, relidas ou inseridas durante a leitura. Nos casos de inserção ou releitura de algumas palavras, os itens inseridos ou relidos foram incorporados ao número total de palavras do texto para o cálculo da taxa. O número de itens omitidos durante a leitura foi subtraído do número total de palavras contidas no texto. Para o cálculo da acurácia identificou-se as palavras lidas corretamente por minuto, considerando-se acerto somente a leitura realizada de maneira correta e fluente. $\mathrm{O}$ mesmo procedimento de cálculo da acurácia na leitura de itens isolados foi utilizado para a leitura dos textos.

As gravações das leituras orais da lista de palavras, ouvidas e transcritas permitiram, adicionalmente, a identificação, a classificação e o cômputo dos totais de erros cometidos por categoria e do total da lista. A análise, categorização e quantificação dos erros nas provas de leitura de palavras foram baseadas em pesquisa anterior ${ }^{(11)}$ considerando as adaptações para o Português Brasileiro a partir das regras de decodificação ortográfica ${ }^{(26)}$. Identificados os erros de leitura, procedeu-se o cálculo do percentual de erros para cada uma das categorias propostas e para o total de erros para a lista de palavras. Para isso identificaramse, a priori, as possibilidades de erros oferecidas pela lista de palavras, considerando-se cada categoria de erros proposta e o total da prova. Considerou-se as seguintes categorias de erros e seus totais de possibilidades de aparecimento:

- T1 - Troca por palavra visualmente similar: quando houve leitura da palavra apresentada como se fosse outra ortograficamente semelhante. (Exemplos: exagero lido como exagerado ou oxítona lida como azeitona). Número de possibilidades de erros oferecidas pelo total de palavras: 38.

- T2 - Regularizações: quando as palavras irregulares foram lidas como regulares (Exemplo: exagero lida como echagero). Total de erros possíveis: 06.

- T3 - Desrespeito à regra de correspondência grafofonêmica independente do contexto: quando se observou a substituição de consoantes, que mantêm relação unívoca com um fonema, ou de vogais durante a leitura de palavras, que ocasionou a leitura incorreta. Exemplo: ganso lida como canso. Total de erros possíveis: 38 .

- T4 - Omissões e adições: de vogais ou consoantes. Total de erros possíveis: 38.

- T5 - Falhas de aplicação de regras ortográficas (Exemplos: mesada lida como messada; gemada lida como guemada). Total de erros possíveis: 30.

- T6 - Inversões de sequência (Exemplo: esgoto lida como egosto). Número total de possibilidades de erros: 39 . 
- T7 - Erro quanto ao emprego da tonicidade: quando houve atribuição correta do valor sonoro dos grafemas, mas erro na identificação da sílaba tônica Exemplo: xale lida como chalé. Número total de possibilidades de erros: 38.

- $\quad$ T8 - Erro por desrespeito ao sinal gráfico de acentuação: quando o sinal de acentuação foi desconsiderado. Exemplo: lâmpada lida como lampada. Número total de possibilidades de erros: 08.

- T9-Erros complexos: quando mais de um erro ocorreu em uma mesma palavra. Número de possibilidades de erros: 38.

- T10 - Recusas: quando a criança se recusou a ler a palavra apresentada. Total de erros possíveis: 38 .

A seguir, calculou-se o índice de produtividade de erros por categoria. Utilizou-se a fórmula:

$\begin{aligned} & \text { Índice percentual de } \\ & \text { produtividade de erros }\end{aligned}=\frac{\begin{array}{l}\text { número de erros cometidos } \\ (\text { por categoria ou no total })\end{array}}{\begin{array}{l}\text { número de erros possíveis } \\ (\text { por categoria ou no total })\end{array}} \times 100$

Todos os dados do GP foram tabulados, e comparados com os do GC. As correlações entre as variáveis foram analisadas em seguida.

\section{RESULTADOS}

Utilizou-se o Programa SPSS (Statistical Package for Social Sciences), em sua versão 10.0. O nível de significância adotado para este estudo foi $5 \%(0,05)$ para todas as análises.

A análise mostrou a presença de diferenças quando compa- radas as frequências de erros, analisados por tipo, apresentados pelos escolares do GP e do GC à leitura da lista de palavras. O GP apresentou mais erros do tipo "T3", “T4”, “T8”, “T9”, "T10" e "Total" que o GC, quando não se levou em conta a série (Tabela 1).

Os dois grupos mostraram-se diferentes quando comparados quanto ao tempo total de leitura, taxa de velocidade e acurácia em todas as provas de leitura realizadas. Observou-se menores valores médios de taxa de velocidade e de acurácia na leitura de palavras, pseudopalavras e textos do GP quando comparado ao GC e maiores valores de tempo despendido pelos escolares do GP na leitura em todas as provas realizadas (Tabela 2).

A comparação realizada por ano escolar mostrou diferença de desempenho para todas as variáveis de fluência estudadas, com exceção de algumas relacionadas à leitura de pseudopalavras, com piores valores dos subgrupos do GP.

Os desempenhos dos dois subgrupos mostraram-se semelhantes na tarefa de leitura de pseudopalavras quando considerados os parâmetros de taxa de velocidade, na comparação entre os escolares de $4^{0}$ e $5^{0}$ anos, e de acurácia, quando os $5^{\circ}$ anos foram comparados (Tabela 3 ).

No GP, notou-se a presença de diferentes correlações. Assim, correlações negativas, de fracas a moderadas, foram encontradas entre T2 com a escolaridade, com a taxa de velocidade na leitura de palavras e de textos e com a acurácia de textos. Correlações negativas e fracas entre T3 e T5 e a escolaridade, e entre o T9 e os valores de acurácia de palavras e de texto, também foram encontradas no GP. O número total de erros correlacionou-se moderada e negativamente com todas as variáveis estudadas (Tabela 4).

Tabela 1. Médias, medianas e desvios-padrão das frequências de erros ortográficos presentes na leitura de palavras dos escolares, distribuídos segundo o grupo e o tipo de erro

\begin{tabular}{|c|c|c|c|c|c|c|c|c|}
\hline Tipo de erro & Grupos & Média & Mediana & $\mathrm{DP}$ & Q1 & Q3 & IC & Valor de $p$ \\
\hline \multirow{2}{*}{ T1 } & GP & 1,56 & 0,0 & 2,33 & 0,0 & 2,6 & 0,84 & \multirow{2}{*}{0,287} \\
\hline & $\mathrm{GC}$ & 1,93 & 2,6 & 2,18 & 0,0 & 2,6 & 0,78 & \\
\hline \multirow{2}{*}{ T2 } & GP & 12,20 & 8,3 & 15,11 & 0,0 & 16,6 & 5,41 & \multirow{2}{*}{$0,078 \#$} \\
\hline & $\mathrm{GC}$ & 3,89 & 0,0 & 7,17 & 0,0 & 0,0 & 2,57 & \\
\hline \multirow{2}{*}{ T3 } & GP & 5,94 & 5,2 & 5,07 & 2,6 & 7,9 & 1,82 & \multirow{2}{*}{$<0,001^{*}$} \\
\hline & GC & 0,70 & 0,0 & 2,07 & 0,0 & 0,0 & 0,74 & \\
\hline \multirow{2}{*}{ T4 } & GP & 4,27 & 2,6 & 3,93 & 2,6 & 5,2 & 1,41 & \multirow{2}{*}{$<0,001^{*}$} \\
\hline & $\mathrm{GC}$ & 0,52 & 0,0 & 1,07 & 0,0 & 0,0 & 0,38 & \\
\hline \multirow{2}{*}{ T5 } & GP & 2,20 & 3,3 & 2,35 & 0,0 & 3,3 & 0,84 & \multirow{2}{*}{0,182} \\
\hline & $\mathrm{GC}$ & 1,44 & 0,0 & 2,26 & 0,0 & 3,3 & 0,81 & \\
\hline \multirow{2}{*}{ T6 } & GP & 0,08 & 0,0 & 0,46 & 0,0 & 0,0 & 0,16 & \multirow{2}{*}{0,531} \\
\hline & $\mathrm{GC}$ & 0,26 & 0,0 & 1,06 & 0,0 & 0,0 & 0,38 & \\
\hline \multirow{2}{*}{ T7 } & GP & 1,65 & 0,0 & 1,99 & 0,0 & 2,6 & 0,71 & \multirow{2}{*}{0,101} \\
\hline & $\mathrm{GC}$ & 2,54 & 2,6 & 2,53 & 0,0 & 4,6 & 0,91 & \\
\hline \multirow{2}{*}{ T8 } & GP & 21,25 & 25,0 & 14,37 & 12,5 & 25,0 & 5,14 & \multirow[t]{2}{*}{$0,036^{*}$} \\
\hline & GC & 13,75 & 12,5 & 11,06 & 0,0 & 25,0 & 3,96 & \\
\hline \multirow{2}{*}{ T9 } & GP & 11,30 & 7,9 & 9,42 & 5,2 & 15,1 & 3,37 & \multirow[t]{2}{*}{$<0,001^{*}$} \\
\hline & $\mathrm{GC}$ & 1,66 & 0,0 & 3,35 & 0,0 & 2,0 & 1,20 & \\
\hline \multirow{2}{*}{ T10 } & GP & 1,40 & 0,0 & 2,56 & 0,0 & 2,6 & 0,91 & \multirow[t]{2}{*}{$0,001^{*}$} \\
\hline & $\mathrm{GC}$ & 0,00 & 0,0 & 0,00 & 0,0 & 0,0 & $-x-$ & \\
\hline \multirow{2}{*}{ Total } & GP & 4,19 & 3,7 & 1,99 & 2,6 & 5,1 & 0,71 & \multirow{2}{*}{$<0,001^{*}$} \\
\hline & $\mathrm{GC}$ & 2,28 & 1,9 & 1,66 & 0,9 & 3,4 & 0,59 & \\
\hline
\end{tabular}

Teste Mann-Whitney; * Valores significativos $(p \leq 0,05)$; \# Valores que tendem à significância estatística

Legenda: $\mathrm{Q} 1=1^{\circ}$ quartil; $\mathrm{Q} 3=3^{\circ}$ quartil; IC = intervalo de confiança; DP = desvio-padrão 
Tabela 2. Distribuição das médias, medianas e desvios-padrão do tempo total, da taxa e da acurácia na leitura de texto, de palavras e de pseudopalavras dos escolares do GP e do GC

\begin{tabular}{|c|c|c|c|c|c|c|c|c|}
\hline Variáveis & Grupos & Média & Mediana & $\mathrm{DP}$ & Q1 & Q3 & IC & Valor de $p$ \\
\hline \multirow{2}{*}{ Tempo(s) palavras } & GP & 93,8 & 88,5 & 35,8 & 64,5 & 121,0 & 12,8 & \multirow{2}{*}{$<0,001^{*}$} \\
\hline & GC & 53,4 & 47,5 & 17,8 & 42,0 & 59,5 & 6,4 & \\
\hline \multirow{2}{*}{ Taxa palavras } & GP & 27,7 & 25,8 & 9,6 & 18,9 & 35,4 & 3,4 & \multirow{2}{*}{$<0,001^{*}$} \\
\hline & GC & 46,9 & 48,0 & 14,0 & 38,3 & 54,3 & 5,0 & \\
\hline \multirow{2}{*}{ Acurácia palavras } & GP & 15,5 & 15,1 & 8,5 & 8,8 & 21,5 & 3,0 & \multirow{2}{*}{$<0,001^{*}$} \\
\hline & GC & 39,9 & 39,0 & 15,0 & 28,6 & 48,0 & 5,4 & \\
\hline \multirow{2}{*}{ Tempo(s) pseudopalavras } & GP & 74,2 & 66,5 & 25,8 & 57,3 & 80,8 & 9,2 & \multirow{2}{*}{$<0,001^{\star}$} \\
\hline & GC & 52,6 & 49,0 & 13,8 & 41,0 & 64,0 & 4,9 & \\
\hline \multirow{2}{*}{ Velocidade pseudopalavras } & GP & 25,6 & 26,2 & 6,7 & 21,6 & 30,5 & 2,4 & \multirow{2}{*}{$<0,001^{*}$} \\
\hline & $\mathrm{GC}$ & 35,3 & 35,5 & 9,1 & 27,2 & 42,4 & 3,3 & \\
\hline \multirow{2}{*}{ Acurácia pseudopalavras } & GP & 14,2 & 14,0 & 6,8 & 9,5 & 17,9 & 2,4 & \multirow{2}{*}{$<0,001^{*}$} \\
\hline & GC & 23,5 & 20,8 & 8,9 & 17,3 & 30,2 & 3,2 & \\
\hline \multirow{2}{*}{ Tempo(s) textos } & GP & 243,5 & 214,5 & 93,5 & 180,8 & 289,0 & 33,5 & \multirow{2}{*}{$<0,001^{*}$} \\
\hline & GC & 119,0 & 114,0 & 46,4 & 91,3 & 135,8 & 16,6 & \\
\hline \multirow{2}{*}{ Velocidade textos } & GP & 50,5 & 50,5 & 17,3 & 38,6 & 61,0 & 6,2 & \multirow{2}{*}{$<0,001^{*}$} \\
\hline & $\mathrm{GC}$ & 107,4 & 106,5 & 32,6 & 87,4 & 128,8 & 11,7 & \\
\hline \multirow{2}{*}{ Acurácia textos } & GP & 45,0 & 41,3 & 18,5 & 32,6 & 58,3 & 6,6 & \multirow{2}{*}{$<0,001^{*}$} \\
\hline & GC & 104,7 & 104,3 & 32,3 & 83,7 & 125,2 & 11,6 & \\
\hline
\end{tabular}

Teste Mann-Whitney; ${ }^{*}$ Valores significativos $(\mathrm{p} \leq 0,05)$

Legenda: (s) = segundos; $\mathrm{Q} 1=1^{\circ}$ quartil; $\mathrm{Q} 3=3^{\circ}$ quartil; IC = intervalo de confiança; $\mathrm{DP}=$ desvio-padrão

O estudo das correlações no GC também mostrou a presença de diferentes correlações negativas, de fracas a moderadas. Assim, T1 correlacionou-se com a taxa de leitura de palavras, de texto e com a acurácia na leitura de texto. Também foram identificadas correlações entre T4 e a acurácia para palavras, T5 e T7 com as variáveis de leitura de texto, T8 e a taxa de leitura de textos, $\mathrm{T} 9$ com a escolaridade e com a taxa de leitura de palavras. O número total de erros correlacionou-se moderada e negativamente com todas as variáveis de fluência (Tabela 5).

\section{DISCUSSÃO}

É importante considerar que esta pequena amostra de escolares foi avaliada, nesta pesquisa, sem que se levassem em conta quaisquer critérios de avaliação clínica. Com o objetivo de caracterizar o GP os desempenhos foram comparados, inicialmente quanto aos tipos de erro ou de imprecisões produzidos na leitura oral de palavras isoladas, sem considerar a variável progressão escolar (Tabela 1). A comparação entre as médias totais de erros diferenciou os dois grupos de escolares, como também puderam observar outros estudos experimentais e revisões da literatura ${ }^{(7,17,19,22)}$.

Adicionalmente, a análise evidenciou que alguns dos tipos de erros de leitura também puderam distinguir o GP do GC. $\mathrm{O}$ desrespeito à regra de correspondência grafo-fonêmica independente do contexto (T3); as omissões e adições (T4); o desrespeito ao sinal gráfico de acentuação (T8); os erros complexos (T9) e as recusas (T10) foram mais frequentes na leitura dos escolares do GP. Considerando-se que a categoria erros complexos abrigou os mesmos tipos de erros definidos desde T1 a T8, o cômputo realizado separadamente por cate- gorias, nesse tipo de erro, certamente, aumentaria a distância entre os desempenhos dos escolares dos dois grupos tornando mais evidentes as dificuldades específicas na leitura do GP. Os valores das médias e dos desvios-padrão, referentes às ocorrências de omissões e adições de letras, e às imprecisões por desrespeito às regras de correspondência direta, podem sugerir que os escolares do GP apresentariam pior desempenho em tarefas de avaliação do processamento da informação fonológica, subjacente à decodificação e reconhecimento de palavras, uma vez que, esses tipos de erro relacionam-se a déficits de processamento fonológico. Estudos realizados com escolares cujas dificuldades de leitura mostravam prejuízos na identificação de grafemas e no reconhecimento de palavras, verificaram maior número de erros e menores potenciais de ativação cortical na leitura de palavras de baixa frequência, concluindo que esses escolares apresentavam déficits em processos sublexicais ${ }^{(7,16,21)}$.

Da mesma forma, as recusas (T10) observadas na leitura do GP, podem ser justificadas pela presença de dificuldades no adequado uso da estratégia de leitura pela rota fonológica, que permitiria, na impossibilidade do reconhecimento de palavras pela via lexical a realização da leitura por conversão grafofonêmica ${ }^{(4)}$. Deve-se considerar, ainda, que a leitura de itens isolados, prova caracterizada pela ausência de informações contextuais, pode ter evidenciado mais facilmente esse tipo de erro no GP. Em estudo anterior, realizado com escolares julgados bons leitores ${ }^{(11)}$, não se encontrou recusas na leitura de palavras isoladas, mesmo nas de baixa frequência de aparecimento.

A análise comparativa dos parâmetros de fluência (Tabela 2) evidenciou diferença entre os Grupos, também, ao se analisarem as taxas de palavras lidas por minuto e de acurácia, re- 
Tabela 3. Distribuição das médias, medianas e desvios-padrão da fluência de leitura dos escolares do $3^{\circ}, 4^{\circ}$ e $5^{\circ}$ ano do GPI, GPII, GPIII, GCI, GCII e GIII distribuídas segundo o tempo total, a taxa e acurácia na leitura de texto, de palavras e de pseudopalavras

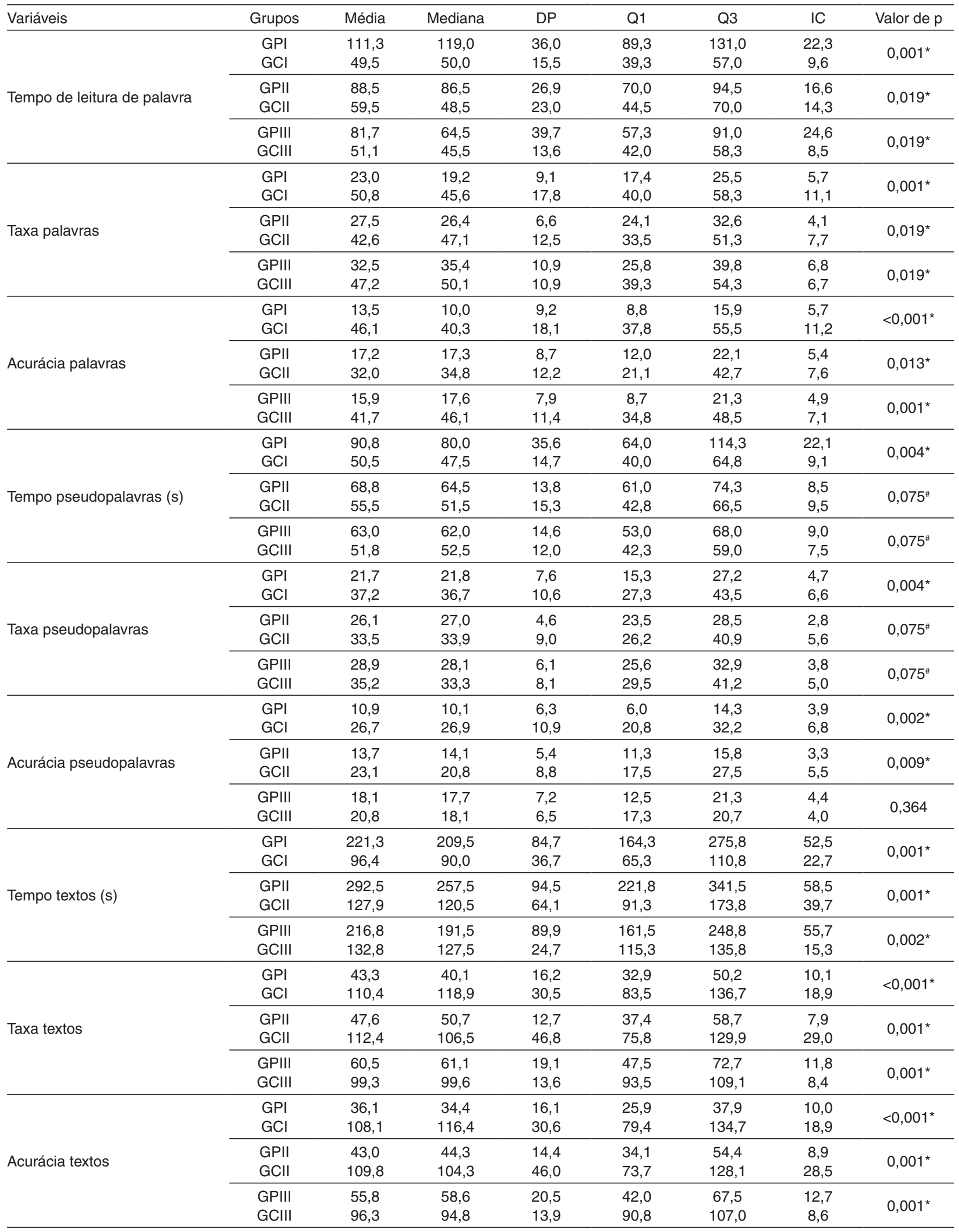


Tabela 4. Correlação do tipo de erros de leitura de palavras dos escolares entre a escolaridade, a taxa de leitura de palavras e de textos e a acurácia de palavras e de textos do GP

\begin{tabular}{|c|c|c|c|c|c|c|}
\hline & & Escolaridade & Taxa_palavras & Acurácia_palavras & Taxa_textos & Acurácia_textos \\
\hline \multirow{2}{*}{ T1_palavras } & Correlação & $16,5 \%$ & $-3,3 \%$ & $-17,4 \%$ & $1,9 \%$ & $-0,6 \%$ \\
\hline & Valor de $p$ & 0,384 & 0,862 & 0,357 & 0,922 & 0,975 \\
\hline \multirow{2}{*}{ T2_palavras } & Correlação & $-38,4 \%$ & $-37,1 \%$ & $-28,8 \%$ & $-46,7 \%$ & $-47,4 \%$ \\
\hline & Valor de $p$ & $0,036^{*}$ & $0,044^{*}$ & 0,123 & $0,009^{*}$ & $0,008^{*}$ \\
\hline \multirow{2}{*}{ T3_palavras } & Correlação & $-36,6 \%$ & $-21,6 \%$ & $-28,4 \%$ & $-25,4 \%$ & $-26,1 \%$ \\
\hline & Valor de $p$ & $0,047^{*}$ & 0,251 & 0,129 & 0,175 & 0,163 \\
\hline \multirow{2}{*}{ T4_palavras } & Correlação & $-25,3 \%$ & $-19,0 \%$ & $-28,5 \%$ & $-19,9 \%$ & $-28,6 \%$ \\
\hline & Valor de $p$ & 0,178 & 0,315 & 0,127 & 0,291 & 0,126 \\
\hline \multirow{2}{*}{ T5_palavras } & Correlação & $-37,7 \%$ & $-26,6 \%$ & $-32,8 \%$ & $-25,4 \%$ & $-28,3 \%$ \\
\hline & Valor de $p$ & $0,040^{*}$ & 0,155 & $0,076 \#$ & 0,175 & 0,129 \\
\hline \multirow{2}{*}{ T6_palavras } & Correlação & $0,0 \%$ & $6,4 \%$ & $20,4 \%$ & $11,8 \%$ & $11,8 \%$ \\
\hline & Valor de $p$ & 1,000 & 0,735 & 0,280 & 0,535 & 0,535 \\
\hline \multirow{2}{*}{ T7_palavras } & Correlação & $30,5 \%$ & $12,7 \%$ & $30,1 \%$ & $20,8 \%$ & $25,3 \%$ \\
\hline & Valor de $p$ & 0,101 & 0,504 & 0,107 & 0,271 & 0,178 \\
\hline \multirow{2}{*}{ T8_palavras } & Correlação & $-18,7 \%$ & $-21,5 \%$ & $-23,9 \%$ & $-30,1 \%$ & $-30,4 \%$ \\
\hline & Valor de $p$ & 0,321 & 0,254 & 0,203 & 0,105 & 0,102 \\
\hline \multirow{2}{*}{ T9_palavras } & Correlação & $-29,4 \%$ & $-27,6 \%$ & $-41,1 \%$ & $-35,4 \%$ & $-39,0 \%$ \\
\hline & Valor de $p$ & 0,115 & 0,140 & $0,024^{*}$ & $0,055 \#$ & $0,033^{*}$ \\
\hline \multirow{2}{*}{ T10_palavras } & Correlação & $-27,5 \%$ & $-25,2 \%$ & $-33,4 \%$ & $-33,3 \%$ & $-31,7 \%$ \\
\hline & Valor de $p$ & 0,142 & 0,179 & $0,071 \#$ & $0,073 \#$ & $0,088^{\#}$ \\
\hline \multirow{2}{*}{ TT_palavras } & Correlação & $-49,7 \%$ & $-48,4 \%$ & $-58,1 \%$ & $-56,2 \%$ & $-60,8 \%$ \\
\hline & Valor de $p$ & $0,005^{*}$ & $0,007^{*}$ & $0,001^{*}$ & $0,001^{*}$ & $<0,001^{*}$ \\
\hline
\end{tabular}

Teste de Correlação de Spearman; * Valores significativos ( $\mathrm{p} \leq 0,05)$; \# Valores que tendem à significância estatística Legenda: correlação fraca $=0$ a 39\%, correlação moderada $=40$ a $79 \%$; correlação forte $=80$ a $100 \%$

Tabela 5. Correlação do tipo de erros de leitura dos escolares entre a escolaridade, a taxa de leitura de palavras e de textos e a acurácia de palavras e de textos do GC

\begin{tabular}{|c|c|c|c|c|c|c|}
\hline & & Escolaridade & Taxa_palavras & Acurácia_palavras & Taxa_textos & Acurácia_textos \\
\hline \multirow[t]{2}{*}{ T1_palavras } & Correlação & $30,7 \%$ & $-40,9 \%$ & $-33,4 \%$ & $-43,7 \%$ & $-45,1 \%$ \\
\hline & Valor de $p$ & $0,098 \#$ & $0,025^{*}$ & $0,072 \#$ & $0,016^{*}$ & $0,012^{*}$ \\
\hline \multirow[t]{2}{*}{ T2_palavras } & Correlação & $29,0 \%$ & $-6,8 \%$ & $-8,7 \%$ & $-10,0 \%$ & $-9,1 \%$ \\
\hline & Valor de $p$ & 0,121 & 0,720 & 0,649 & 0,598 & 0,632 \\
\hline \multirow[t]{2}{*}{ T3_palavras } & Correlação & $0,0 \%$ & $-3,8 \%$ & $-8,6 \%$ & $1,1 \%$ & $-0,2 \%$ \\
\hline & Valor de $p$ & 1,000 & 0,842 & 0,650 & 0,954 & 0,994 \\
\hline \multirow[t]{2}{*}{ T4_palavras } & Correlação & $-10,2 \%$ & $-34,7 \%$ & $-36,6 \%$ & $-28,9 \%$ & $-27,9 \%$ \\
\hline & Valor de $p$ & 0,591 & $0,060 \#$ & $0,047^{\star}$ & 0,122 & 0,135 \\
\hline \multirow[t]{2}{*}{ T5_palavras } & Correlação & $15,3 \%$ & $-7,2 \%$ & $-23,2 \%$ & $-43,4 \%$ & $-40,2 \%$ \\
\hline & Valor de $p$ & 0,419 & 0,706 & 0,217 & $0,017^{*}$ & $0,028^{*}$ \\
\hline \multirow[t]{2}{*}{ T6_palavras } & Correlação & $-15,8 \%$ & $-28,3 \%$ & $-23,9 \%$ & $-9,0 \%$ & $-9,0 \%$ \\
\hline & Valor de $p$ & 0,404 & 0,130 & 0,204 & 0,638 & 0,638 \\
\hline \multirow[t]{2}{*}{ T7_palavras } & Correlação & $9,0 \%$ & $-33,6 \%$ & $-33,8 \%$ & $-54,9 \%$ & $-54,4 \%$ \\
\hline & Valor de $p$ & 0,636 & $0,070 \#$ & $0,068 \#$ & $0,002^{*}$ & $0,002^{*}$ \\
\hline \multirow[t]{2}{*}{ T8_palavras } & Correlação & $9,9 \%$ & $1,7 \%$ & $-22,4 \%$ & $-39,7 \%$ & $-34,6 \%$ \\
\hline & Valor de $p$ & 0,601 & 0,930 & 0,234 & $0,030^{*}$ & $0,061 \#$ \\
\hline \multirow[t]{2}{*}{ T9_palavras } & Correlação & $-42,8 \%$ & $-37,2 \%$ & $-18,2 \%$ & $-8,6 \%$ & $-10,6 \%$ \\
\hline & Valor de $p$ & $0,018^{*}$ & $0,043^{*}$ & 0,337 & 0,653 & 0,579 \\
\hline \multirow[t]{2}{*}{ T10_palavras } & Correlação & - & - & - & - & - \\
\hline & Valor de $p$ & - & - & - & - & - \\
\hline \multirow[t]{2}{*}{ TT_palavras } & Correlação & $-0,7 \%$ & $-53,6 \%$ & $-53,3 \%$ & $-58,7 \%$ & $-58,2 \%$ \\
\hline & Valor de $p$ & 0,970 & $0,002^{*}$ & $0,002^{*}$ & $0,001^{*}$ & $0,001^{*}$ \\
\hline
\end{tabular}

Teste de Correlação de Spearman; * Valores significativos $(p \leq 0,05)$; \# Valores que tendem à significância estatística

Legenda: $x=$ quando não foi possível utilizar a estatística; correlação fraca = 0 a 39\%, correlação moderada = 40 a $79 \%$; correlação forte $=80$ a $100 \%$ 
velando pior desempenho do GP. Esses resultados corroboram os de outros estudos que observaram desempenhos superiores de bons leitores quando comparados a maus leitores, quanto à fluência de leitura ${ }^{(3,20,23)}$. A literatura vem indicando que a automaticidade, reconhecimento rápido e acurado de palavras, é fundamentalmente uma característica de bons leitores, permitindo, assim, mais facilmente, o desenvolvimento e alcance da leitura com compreensão ${ }^{(27)}$. Estudos mostram que escolares com dificuldades de leitura apresentam maiores dificuldades ao lerem itens isolados e textos, que os pares sem dificuldades, nos anos iniciais da escolaridade ${ }^{(7,18)}$.

Outras pesquisas realizadas com escolares com dificuldades ou transtornos, observaram melhora de desempenho com o avanço dos anos escolares ${ }^{(3,24)}$, evidenciando progressiva apropriação das regras de decodificação ortográfica do Português Brasileiro mesmo nesses grupos. Quando se observam os valores de taxa e acurácia na leitura de textos do GP pode-se constatar esse progresso (Tabela 3 ).

Deve-se, considerar, nessa análise comparativa que houve semelhança de desempenhos dos subgrupos quando estudados os parâmetros de taxa de leitura de pseudopalavras por minuto, na comparação entre GPII e GCII ( $4^{\circ}$ ano) e entre GPIII e GCIII ( $5^{\circ}$ ano), e de acurácia, quando os quintos anos foram comparados. Os valores das médias de acurácia, velocidade e tempo na leitura de pseudopalavras pelo GP evidenciaram certa evolução do GP, provavelmente relacionada à melhora da automaticidade e à possibilidade de diminuição de erros com a seriação, pelo aumento de utilização dos processos de decodificação expressos, nesta pesquisa, pela melhora progressiva da leitura de pseudopalavras, como sinal de desenvolvimento da rota fonológica de leitura, conforme avançou a escolaridade ${ }^{(3,24)}$ (Tabela 3). Porém, é importante mencionar que a desconsideração de possíveis atendimentos terapêuticos no caso dos escolares do GP pode ter influenciado os resultados dessa análise.

Nesta pesquisa, também se investigou a presença de correlações entre escolaridade, tipos de erros e parâmetros de fluência de leitura, separadamente, para cada grupo. No estudo do GP alguns tipos de erros mostraram que suas ocorrências foram tanto mais frequentes, quanto mais inicial foi o ano escolar estudado (Tabela 4). A maior frequência de ocorrência desses erros também se associou aos mais baixos valores de taxa de leitura e de acurácia e a mais tempo despendido para a leitura dos diferentes itens. Assim, correlações desse tipo foram observadas entre o erro de regularização (T2), que envolve o conhecimento de decodificação ortográfica da letra $\mathrm{x}$, e a escolaridade, taxas de velocidade e de acurácia na leitura de palavras e texto. Ou seja, quanto mais inicial a escolaridade, mais frequentemente os escolares do GP produziram erros de regularização e despenderam mais tempo para ler. Neste caso, pode-se pensar a possibilidade de interpretar o erro como estratégia, ou tentativa de acerto, quando se admite que quanto mais inicial o ano escolar mais se utilizou a estratégia fonológica de leitura para itens que apresentaram, por exemplo, a necessidade de decodificação da letra x, cuja representação no léxico ortográfico depende da experiência leitora e do aprendizado formal da regra. O mesmo foi observado em relação à categoria erros complexos (T9) que mostrou correlação negativa com a taxa de velocidade de textos e de acurácia de leitura de pseudopalavras e palavras, dificultando a leitura e levando o escolar a despender mais tempo na tarefa.

Quando se investigou a presença de outras correlações entre a escolaridade e os erros no GP, pôde-se observar que o desrespeito à regra de correspondência fonema-grafema (T3), a falha de aplicação de regras dependentes do contexto (T5) e, até mesmo o número total de erros (TT) correlacionaram-se negativamente com a progressão escolar. Ou seja, quanto mais adiantado o ano escolar investigado, menor a média desse tipo de erros. Apesar das diferenças quando comparados ao GC, a escolaridade ou a instrução formal parecem determinantes para o progresso do aprendizado da leitura e das regras ortográficas para a decodificação e o reconhecimento automático de palavras. Estas correlações, apesar de fracas e moderadas corroboram os achados de outro estudo que também observou redução de erros, conforme o aumento da fluência de leitura de palavras e a escolaridade ${ }^{(28)}$.

No GP, o número total de erros (TT) correlacionou-se negativa e moderadamente com todos os parâmetros de fluência e com a escolaridade, resultado que mostra a influência da decodificação ortográfica sobre parâmetros de fluência da leitura, principalmente nesses três anos escolares, em crianças com queixas e indicações de dificuldade leitora.

No GP, a tentativa ou o fracasso de ler corretamente influenciou os parâmetros de fluência, tanto mais frequentemente, quanto mais inicial foi o ano escolar. Os achados revelaram que as dificuldades em lidar com as irregularidades da língua e o grande número de erros na decodificação foram os fatores que prejudicaram, significativamente, a fluência de leitura desse grupo de escolares.

No grupo, de escolares sem indícios de dificuldades (GC), observou-se padrão diferente de correlações quanto aos tipos de erro. Observou-se correlações entre as trocas por palavra visualmente similar (T1) com as taxas de velocidade na leitura de palavras e de velocidade e acurácia na leitura de textos; as omissões e adições (T4) com a taxa de acurácia na leitura de palavras; as falhas de aplicação de regras ortográficas dependentes do contexto (T5) e o emprego da tonicidade (T7) com os parâmetros de leitura de texto; os erros por desrespeito ao sinal gráfico de acentuação (T8) com a taxa de velocidade da leitura de texto; e os erros complexos (T9) com a escolaridade e a taxa de velocidade de leitura de palavras.

As correlações observadas para o GC sugerem que a redução dos erros relacionados ao processamento fonológico (omissões e adições) e ao mau uso das estratégias lexicais (erros de substituição da palavra alvo por outra visualmente similar ou falha das regras de aplicação ortográficas dependentes do contexto) concorreram para o aumento da taxa de velocidade de leitura de palavras e de texto, e a acurácia de palavras e de textos, melhorando assim a automaticidade durante a decodificação de leitura. As explicações para estes resultados sustentam-se em estudos que também encontraram redução de erros, conforme o melhor desempenho na leitura e maior porcentagem de acertos no reconhecimento de palavras ${ }^{(4,20,24,28)}$.

Também no GC observou-se que a progressão das séries escolares correlacionou-se à diminuição da frequência de ocorrência de erros complexos na leitura de itens isolados dos escolares sem queixas, o que não foi possível observar 
no GP. Este resultado é similar aos encontrados por outros pesquisadores $^{(16,29,30)}$.

Assim, as correlações encontradas para cada grupo investigado mostraram diferentes padrões de ocorrência. $\mathrm{O}$ confronto desses diferentes padrões permite caracterizar os grupos com e sem dificuldades de leitura. Como exemplo, pode-se verificar no GP, que o desrespeito à regra de correspondência fonemagrafema (T3) influenciou a taxa e, consequentemente o tempo total despendido na leitura de texto, enquanto no GC, as trocas por palavra visualmente similar (T1) associaram-se a piores valores de fluência da leitura dos escolares. Esses resultados sugerem que apesar da diversidade entre as capacidades de leitura envolvidas na produção de cada um desses dois tipos de erro, ambas influenciaram em cada grupo, o número de palavras lidas por minuto e a precisão com que essas palavras puderam ser lidas. Apesar das limitações, impostas pelo tamanho da amostra e pela falta de investigações complementares sobre outras habilidades envolvidas nos mecanismos de leitura ou na capacidade de compreensão, os resultados desta pesquisa sugerem que a análise dos tipos de erros produzidos pelos escolares durante a leitura de palavras pode fornecer informações para a caracterização de outras variáveis da leitura. Além disso, permitem supor que, até o momento das avaliações, ambos os grupos desenvolveram diferentes estratégias de decodificação e leitura.

Por fim, o desempenho dessa população de escolares da rede pública com e sem queixas, pareados segundo idade e escolaridade, mostrou, também, a possibilidade de utilização dos parâmetros de fluência e das categorias de erros de leitura como indicadores de dificuldades no aprendizado da leitura e da escrita. Os resultados apontam ainda a possibilidade de se estabelecer, a partir de estudos futuros, dados normativos de desempenho para cada um destes parâmetros, o que forneceria balizadores para um diagnóstico da situação dos escolares brasileiros quanto às capacidades leitoras.

\section{CONCLUSÃO}

A partir das análises comparativas dos desempenhos do GP e do GC, foi possível concluir que os escolares com indícios de dificuldades de leitura e de escrita diferenciaram-se dos escolares indicados como bons leitores, de mesma idade e ano escolar, por apresentarem maior número de erros e menores valores médios de taxa e acurácia de leitura de palavras, pseudopalavras e textos. Dentre os tipos de erros cometidos, observou-se que o GP diferenciou-se especialmente, por apresentar maior número de erros complexos e de erros relacionados a dificuldades no processamento fonológico: desrespeito à regra de correspondência grafo-fonêmica independente do contexto, omissões e adições e recusas para ler. Erros por desrespeito ao sinal gráfico de acentuação gráfica também diferenciaram os grupos, mas com menor significância. A melhora do GP manifestou-se na análise comparativa da leitura de pseudopalavras nos quartos e quintos anos, que revelou similaridade no desempenho dos grupos.

As análises obtidas neste estudo fornecem evidências importantes para o contexto educacional brasileiro e para a prática clínica. No contexto educacional, a observação da progressão da apropriação ortográfica ao longo das séries, bem como sinais de disparidade no desempenho da fluência leitora de um dado escolar, em comparação aos colegas de sala, pode servir de alerta precoce ao professor sobre riscos para o aprendizado da leitura e sobre a necessidade de encaminhamentos para investigação clínica das manifestações. Estudos futuros podem estabelecer dados normativos de desempenho quanto aos parâmetros de fluência e de análise dos erros de leitura, em função da seriação escolar, fornecendo balizadores para o diagnóstico pedagógico/educacional e clínico/fonoaudiológico nos casos em que as dificuldades sejam determinadas pela presença de reais distúrbios ou transtornos.

\section{AGRADECIMENTOS}

À Fundação de Amparo à Pesquisa do Estado de São Paulo - FAPESP, pela bolsa concedida para realização desta pesquisa, sob processo n ${ }^{\circ}$ 2008/57154-1.

\begin{abstract}
Purpose: To characterize the performance of students with signs of reading and writing difficulties, according to the school grade, error categories, reading fluency parameters, and the correlation between these variables. Methods: Sixty children (48\% girls), $3^{\text {rd }}$ to the $5^{\text {th }}$ grade students of public elementary schools, were evaluated. Thirty (ten from each grade) who presented signs of reading and writing difficulties composed the Research Group. Thirty children, paired by age and school grade, classified by their teachers as good readers, composed the Control Group. All subjects read aloud two lists of isolated items (38 words and 29 pseudowords) and a text. The reading sessions were recorded and transcribed, and parameters and errors were analyzed. Results: Differences were found between the groups, and the Research Group had worse performances in all the studied variables. The following types of reading errors were more frequent in this group: non-compliance with the context-independent matching rule, omissions and additions, noncompliance with stress, complex errors, and refusals. Fluency rates and values were lower in the students with reading complaints, when compared to the good readers. Negative correlations were identified between reading fluency variables and the different types of errors, with different correlation values for each group, and showed that, in the present sample, the total number of errors decreased with school progression. Conclusion: The students with signs of reading and writing difficulties had worse reading fluency performance, and higher number of errors in all the grades studied. The correlations found evidenced the influence of the type of error on reading fluency, according to different patterns for each group.
\end{abstract}

Keywords: Evaluation; Dyslexia; Educational status; Learning disorders; Comprehension 


\section{REFERÊNCIAS}

1. Brasil. Ministério da Educação. Instituto Nacional de Estudos e Pesquisas Educacionais Anísio Teixeira. Programa Internacional de Avaliação de Alunos - PISA. [Internet]. Disponível em: http://www. inep.gov.br/internacional/pisa/ .

2. Fuchs LS, Fuchs D, Hosp M, Jenkins JR. Oral reading fluency as an indicator of reading competence: a theoretical, empirical, and historical analysis. Sci Stud Read. 2001;5(3):239-56.

3. Salles JF, Parente MAMP. Processos cognitivos na leitura de palavras em crianças: relações com compreensão e tempo de leitura. Psicol Reflex Crít. 2002;15(2):321-31.

4. Pinheiro AMV, Cunha CR, Lúcio OS. Tarefa de leitura de palavras em voz alta: uma proposta de análise dos erros. Rev Port Educ. 2008;21(2):115-38.

5. Mann VA. Longitudinal prediction and prevention of early reading difficulty. Ann Dyslexia. 1984;34:117-36.

6. Nation K, Snowling MJ. Semantic processing and the development of word-recognition skills: evidence from children with reading comprehension difficulties. J Mem Lang. 1998;39(1):85-101.

7. Capellini SA, Cavalheiro LG. Avaliação do nível e da velocidade de leitura em escolares com e sem dificuldade na leitura. Temas Desenvolv. 2000;9(51):5-12.

8. Geva E, Yaghoub Zadeh Z. Reading efficiency in native Englishspeaking and English-as-a-second-language children: the role of oral proficiency and underlying cognitive-linguistic processes. Sci Stud Read. 2006;10(1):31-57.

9. Schwanenglugel PJ, Hamilton AM, Wisenbaker JM, Kuhn MR, Stahl SA. Becoming a fluent reader: reading skill and prosodic features in the oral reading of young readers. J Educ Psychol. 2004;96(1):119-29.

10. Alves LM, Pinheiro AMV, Reis C, Capellini SA. Medidas objetivas de fluência de leitura e o processo de compreensão. In: Barbosa T, Rodrigues CC, Mello CB, Capellini SA, Mousinho R, Alves LM, organizadores. Temas em dislexia. São Paulo: Artes Médicas; 2009. p. 89-102.

11. Avila CRB, Kida ASB, Carvalho CAF, Paolucci JF. Tipologia de erros de leitura de escolares brasileiros considerados bons leitores. Pró-Fono. 2009;21(4):320-5.

12. Tannenbaum KR, Torgesen JK, Wagner RK. Relationships between word knowledge and reading comprehension in third-grade children. Sci Stud Read. 2006;10(4):391-8.

13. Rapp DN, van den Broek P, McMaster KL, Kendeou P, Espin CA. Higher-order comprehension processes in struggling readers: a perspective for research and Intervention. Sci Stud Read. 2007;11(4):289-312.

14. Donald DR. Analysis of children's oral reading errors: a current perspective. J Res Read. 1980;3(2):106-14.
15. Goikoetxea E. Reading errors in first- and second-grade readers of a shallow ortography: evidence from Spanish. Br J Educ Psychol. 2006;76(Pt 2):333-50.

16. Fox E. Grapheme-phoneme correspondence in dyslexic and matched control readers. Br J Psychol. 1994;85(Pt 1):41-53.

17. Capovilla AGS, Capovilla FC, Suiter I. Processamento cognitivo em crianças com e sem dificuldades de leitura. Psicol Estud. 2004;9(3):44958.

18. Capovilla AGS, Joly MCRA, Ferracini F, Caparrotti NB, Carvalho MR, Raad AJ. Estratégias de leitura e desempenho em escrita no início da alfabetização. Psicol Esc Educ. 2004;8(2):189-97.

19. Ramos CS. Avaliação da leitura em escolares com indicação de dificuldades de leitura e escrita [dissertação]. São Paulo: Universidade Federal de São Paulo. Escola Paulista de Medicina; 2005.

20. Tonelotto JMF, Fonseca LC, Tedrus GMSA, Martins SMV, Gibert MAP, Antunes TA, Pensa NAS. Avaliação do desempenho escolar e habilidades básicas de leitura em escolares do ensino fundamental. Aval Psicol. 2005;4(1):33-43.

21. Paul I, Bott C, Wienbruch C, Elbert TR. Word Processing differences between dyslexic and control children. BMC Psychiatry. 2006;6:5

22. Capellini AS, Conrado TLBC. Desempenho de escolares com e sem dificuldades de aprendizagem de ensino particular em habilidade fonológica, nomeação rápida, leitura e escrita. Rev CEFAC. 2009;11(Supl 2):183-93.

23. Carvalho CAF, Ávila CRB, Chiari BM. Níveis de compreensão de leitura em escolares. Pró-Fono. 2009;21(3):207-12.

24. Grainger J, Bouttevin S, Truc C, Bastien M, Ziegler J. Word superiority, pseudoword superiority, and learning to read: a comparison of dyslexic and normal readers. Brain Lang. 2003;87(3):432-40.

25. Kida ASB. Bateria de avaliação de habilidades e competências linguísticas, de escrita e de leitura: estudo piloto [tese]. São Paulo: Universidade Federal de São Paulo. Escola Paulista de Medicina; 2009.

26. Scliar-Cabral L. Princípios do sistema alfabético do português do Brasil. São Paulo: Contexto; 2003.

27. LaBerge D, Samuels SJ. Toward a theory of automatic information processing in reading. Cognit Psychol. 1974;6(2):293-323.

28. Kingeski MF, Sisto FF. Reconhecimento de palavras nas séries iniciais do ensino fundamental. Teor Prat Educ. 2004;7(2):183-4.

29. Stivanin L, Scheuer CI. Tempo de latência e exatidão para leitura e nomeação em crianças escolares: estudo piloto. Educ Pesqui. 2005;31(3):425-36.

30. Cunha VLO, Capellini SA. Desempenho de escolares de $1^{a}$ a $4^{a}$ série do ensino fundamental nas provas de habilidades metafonológicas e de leitura - PROHMELE. Rev Soc Bras Fonoaudiol. 2009;14(1):56-68. 